\title{
Improvement of parameter estimation for non-linear hysteretic systems with slip by a fast Bayesian bootstrap filter
}

\author{
S.J. Li ${ }^{\mathrm{a}, *}$, Y. Suzuki ${ }^{\mathrm{b}}$, M. Nooric \\ ${ }^{a}$ Institute of Civil Engineering and Architecture, Wuhan University of Technology, Wuhan 430070, PR China \\ ${ }^{\mathrm{b}}$ Disaster Prevention Research Institute, Kyoto University, Uji, Kyoto 611-0011, Japan \\ ${ }^{\mathrm{c}}$ Department of Mechanical and Aerospace Engineering, North Carolina State University, NC 27695, USA
}

\begin{abstract}
Modeling and identification of non-linear hysteretic systems are widely encountered in the structural dynamics field, especially for the hysteresis with slip. A model, called SL model, which can describe the pinching of most practical hysteresis loops perfectly was proposed by Baber and Noori (J. Eng. Mech. 111 (1985) 1010). A method of estimating the parameters of SL model on the basis of input-output data based on bootstrap filter was proposed by the writers. Bootstrap filter is a filtering method based on Bayesian state estimation and Monte Carlo method, which has the great advantage of being able to handle any functional non-linearity and system and/or measurement noise of any distribution. The standard bootstrap filter, however, is not time efficient, i.e., it is very time consuming and is not suitable for real-time applications. In this paper, previous work by the writers is extended to do the parameter estimation of SL model by a fast Bayesian bootstrap filtering technique. Simulation results are presented to demonstrate the performance of the algorithm.
\end{abstract}

Keywords: System identification; Hysteretic system; Bootstrap filter; Bayesian estimation; Monte Carlo method

\section{Introduction}

Many civil engineering structures exhibit hysteresis when subjected to severe dynamic loading, i.e., the restoring force of structure depends not only on the instantaneous deformation but also on the past history of deformation. As a result, the hysteretic restoring force cannot be expressed by an algebraic function of the instantaneous displacement and velocity. This memory nature renders the hysteretic systems more

\footnotetext{
* Corresponding author. Tel.: +86-27-873-83401; fax: +86-27876-51992.

E-mail address: sjli@mail.whut.edu.cn (S.J. Li).
}

difficult to model and analyze than other non-linear systems, especially for the hysteresis with slip. Modeling and identification of these hysteretic systems are of considerable theoretical and practical interest over the years because of its importance in response prediction, structural control and health monitoring.

Much effort has been devoted by numerous investigators to develop models of hysteretic systems [1]. One of the widely accepted model is a differential model originally proposed by Bouc [2] and further developed and generalized by Wen and his colleagues $[3,4]$. In this model the restoring force and the deformation are connected through a non-linear differential equation containing unspecified parameters. By choosing the parameters suitably, it is possible to generate 
a large variety of different shapes of the hysteresis loops. Based on the Bouc-Wen model, a smoothed hysteretic slip model, which can describe the pinching of hysteresis loops perfectly was proposed by Baber and Noori [5]. The model, called SL model, consists of a non-pinching hysteretic element in series with a 'slip-lock' element and contains six loop parameters.

System identification refers to any systematic way of deriving or improving models through the use of experimental data. System identification techniques can be classified into parametric and non-parametric procedures. In the parametric identification procedure, the mathematical model of the system is a priori known from theoretical considerations, and only the values of model parameters are to be determined from experimental data. On the other hand, the non-parametric identification procedure does not require a priori knowledge of system model, but instead yields the best representation of the system in the form of a multiple-indexed series of functions or functionals [6,7]. As a parametric identification procedure, the method based on extended Kalman filter (EKF) has received much attention and has been used successfully in the parameter estimation problems over the past years $[8,9]$. In this procedure, the estimation problem is linearized about the predicted state so that the Kalman filter can be applied. However, the linearization process and the assumption of a Gaussian probability density function (PDF) may be a gross distortion of the true underlying structure and may lead to estimation divergence.

A filtering method, called bootstrap filter, based on Bayesian state estimation and Monte Carlo method was proposed by Gordon [10]. The idea of this method is to represent the required PDF as a set of random samples, rather than as a function over state space. As the number of samples becomes large, they can effectively provide an accurate representation of the required PDF. Estimations of states can then be obtained directly from the samples. The advantage of this approach over the EKF is that no linearization is needed and the required PDFs are not restricted to Gaussian. Thus, it is particularly well suited for the identification of complicated non-linear systems. Using this filtering technique, a method to identify parameters of non-linear and non-Gaussian models subjected to dynamic forces was proposed by Hoshiya [11].
In the past decades, the parametric identification of non-linear hysteretic systems of differential type models has been extensively studied [12-15], but mostly for the Bouc-Wen model or other linear hysteretic models. There are seldom papers on the estimation of hysteretic systems with slip because of its complexity.

A parametric identification method for the SL model based on bootstrap filter was proposed by the writers [16]. However, a drawback of the method is the heavy computational load, which precludes its utilization for real time estimations. A fast Bayesian bootstrap filter, which can dramatically decreases the computation time of the standard bootstrap filter, was proposed by Beadle and Djuric [17]. The time decrease is realized by resampling the prior into the posterior distribution at each time step by using sampling groups, rather than a sample as in the standard approach. The groups are generated based on the expected number of times at which each value in the prior is resampled to the posterior. Using this filtering technique, a new algorithm is proposed in the present paper for estimating parameters in the SL model. The new algorithm is in fact an improvement of the previous work by the writers [16]. A simulation is performed for an example for comparing the two algorithms.

\section{Hysteretic slip model}

In this section we briefly present the SL model proposed by Baber and Noori [5]. More details about this model are given in $[5,16]$. This differential model to describe hysteretical system with slip is governed by

$$
\begin{aligned}
& \ddot{x}+2 \zeta \omega_{0} \dot{x}+\alpha \omega_{0}^{2} x+(1-\alpha) \omega_{0}^{2} \bar{z}=p(t), \\
& \dot{\bar{z}}=\bar{A} \dot{x}_{1}-\bar{\beta}\left|\dot{x}_{1}\right| \bar{z}|\bar{z}|^{n-1}-\bar{\gamma} \dot{x}_{1}|\bar{z}|^{n}, \\
& \dot{x_{2}}=\sqrt{\frac{2}{\pi}} \frac{s}{\sigma} \exp \left[-\frac{\bar{z}^{2}}{2 \sigma^{2}}\right] \dot{\bar{z}}, \\
& x=x_{1}+x_{2}
\end{aligned}
$$

in which $p(t)$ is the input, $\alpha$ is the ratio of post-yield/pre-yield stiffness, and $\bar{z}$ is the hysteretic displacement. $\bar{A}, \bar{\beta}, \bar{\gamma}$ and $n$ are model parameters. Among the parameters, $\bar{\beta}, \bar{\gamma}$ and $n$ determine the hysteresis shape, and $\bar{A}$ determines the tangent stiffness. The hysteresis loop pinching is added by incorporating a time-dependent 'slip-lock' element as shown 


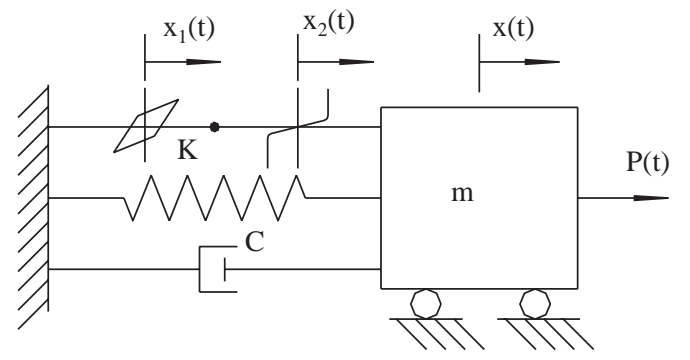

Fig. 1. Hysteretic slip model.

in Figs. 1 and 2, and the relationship between the 'slip-lock' and hysteretic displacement is described by Eq. (3) where $s$ is the magnitude of slip, and $\sigma$ reflects the degree of pinching and sharpness of hysteresis loops, as shown in Fig. 3.

By defining a set of new parameters as follows:

$a=2 \zeta \omega_{0}, \quad b=\alpha \omega_{0}^{2}$,

$z=(1-\alpha) \omega_{0}^{2} \bar{z}, \quad A=(1-\alpha) \omega_{0}^{2} \bar{A}$,

$\beta=\bar{\beta} /\left[(1-\alpha) \omega_{0}^{2}\right]^{n-1}, \quad \gamma=\bar{\gamma} /\left[(1-\alpha) \omega_{0}^{2}\right]^{n-1}$,

Eqs. (1)-(4) can be rewritten as

$\ddot{x}+a \dot{x}+b x+z=p(t)$,

$\dot{z}=A \dot{x}_{1}-\beta\left|\dot{x}_{1}\right| z|z|^{n-1}-\gamma \dot{x}_{1}|z|^{n}$,

$\dot{x}_{2}=\sqrt{\frac{2}{\pi}} \frac{s}{\sigma} \exp \left[-\frac{z^{2}}{2 \sigma^{2}}\right] \dot{z}$,

$x=x_{1}+x_{2}$.

In general, the magnitude of slip $s$ may vary with the history of response. In this model $s$ is assumed

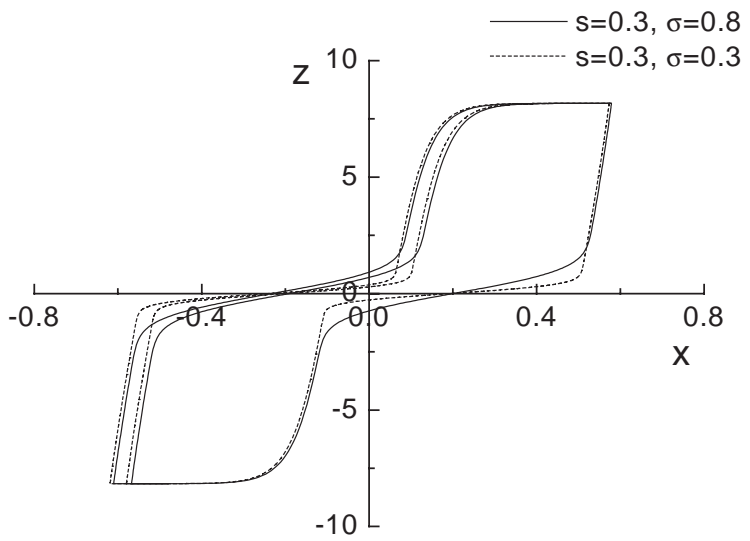

Fig. 3. Pinching behavior of model under different $\sigma$.

to be a function of the system energy dissipation $E(t)$ as follows:

$s=\delta_{s} E(t)$,

where $\delta_{s}$ is a constant. Eqs. (5)-(9) complete the model, and the parameters need to be identified are $a$, $b, A, \beta, \gamma, n, \delta_{s}$ and $\sigma$.

\section{Fast Bayesian bootstrap filter}

In this section, both the original bootstrap filter [10] and the fast Bayesian bootstrap filter [17] will be reviewed briefly.

\subsection{Bootstrap filter}

The system model is assumed to have the discrete form

$x_{k}=f\left(x_{k-1}, w_{k}\right)$,

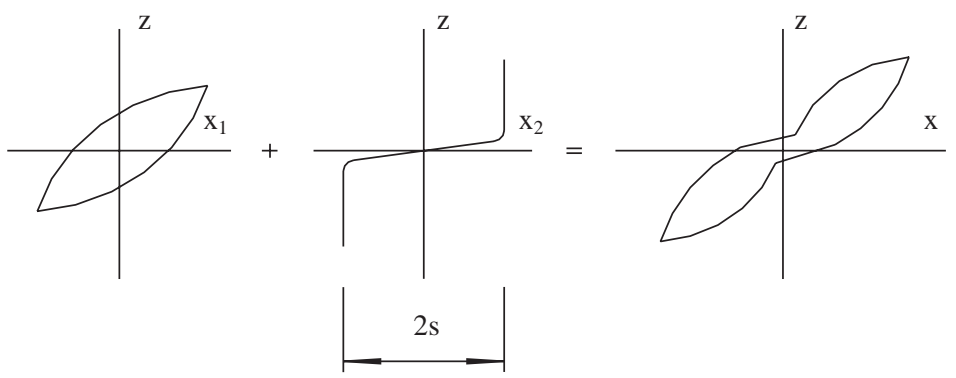

Fig. 2. Slip-lock series hysteresis. 
where $f: R^{n} \times R^{m} \rightarrow R^{n}$ is the system transition function and $w_{k} \in R^{m}$ is a zero-mean noise process independent of the system states. The PDF of $w_{k}$ is assumed to be known as $p_{w}\left(w_{k}\right)$. At discrete times, measurements are denoted by $y_{k} \in R^{p}$, which are related to the state vector via the observation equation

$y_{k}=h\left(x_{k}, v_{k}\right)$,

where $h: R^{n} \times R^{r} \rightarrow R^{p}$ is the measurement function, and $v_{k} \in R^{r}$ is the observation noise, assumed to be another zero mean random sequence independent of both state variable $x_{k}$ and the system noise $w_{k}$. The PDF of $v_{k}$ is assumed to be known as $p_{v}\left(v_{k}\right)$. The available information at time step $k$ is the set of measurements $D_{k}=\left\{y_{i}: i=1, \ldots, k\right\}$. It is noted that $f\left(x_{k-1}, w_{k}\right)$ and $h\left(x_{k}, v_{k}\right)$ are generally not linear functions, and $x_{k}, w_{k}$ and $v_{k}$ are not necessarily Gaussian vectors.

It is required to construct the PDF of the current state $x_{k}$, given all the available information: $p\left(x_{k} \mid D_{k}\right)$. In principle, this PDF may be obtained recursively in two stages: prediction and update. Suppose that the required PDF $p\left(x_{k-1} \mid D_{k-1}\right)$ at time step $k-1$ is available. Then using the system model it is possible to obtain the prior PDF of the state at time step $k$

$$
\begin{aligned}
p\left(x_{k} \mid D_{k-1}\right)= & \int p\left(x_{k} \mid x_{k-1}\right) \\
& \times p\left(x_{k-1} \mid D_{k-1}\right) \mathrm{d} x_{k-1},
\end{aligned}
$$

where $p\left(x_{k} \mid x_{k-1}\right)$ is determined by $f\left(x_{k-1}, w_{k}\right)$ and the distribution of $w_{k}$ in system equation (10). Then at time step $k$, a measurement $y_{k}$ becomes available and may be used to update the prior according to the Bayes' rule

$p\left(x_{k} \mid D_{k}\right)=\frac{p\left(y_{k} \mid x_{k}\right) p\left(x_{k} \mid D_{k-1}\right)}{\int p\left(y_{k} \mid x_{k}\right) p\left(x_{k} \mid D_{k-1}\right) \mathrm{d} x_{k}}$,

where the conditional PDF $p\left(y_{k} \mid x_{k}\right)$ is determined from the measurement model and the known statistics of $v_{k}$

$p\left(y_{k} \mid x_{k}\right)=\int \delta\left(y_{k}-h\left(x_{k}, v_{k}\right)\right) p_{v}\left(v_{k}\right) \mathrm{d} v_{k}$.

The bootstrap filter is a recursive algorithm to estimate the posterior $p\left(x_{k} \mid D_{k}\right)$ from a set of samples
[10]. Assuming that we have a set of random samples $\left\{x_{k-1}(i): i=1, \ldots, m\right\}$ from the PDF $p\left(x_{k-1} \mid D_{k-1}\right)$, the filter procedure is as follows:

(1) Prediction: Each sample from PDF $p\left(x_{k-1} \mid\right.$ $\left.D_{k-1}\right)$ is passed through the system model to obtain samples from the prior at time step $k$ :

$x_{k}^{*}(i)=f\left(x_{k-1}(i), w_{k}(i)\right)$,

where $w_{k}(i)$ is a sample drawn from the PDF of the system noise $p_{w}\left(w_{k}\right)$.

(2) Update: On receipt of the measurement $y_{k}$, evaluate the likelihood of each prior sample and obtain the normalized weight for each sample

$q_{i}=\frac{p\left(y_{k} \mid x_{k}^{*}(i)\right)}{\sum_{j=1}^{m} p\left(y_{k} \mid x_{k}^{*}(j)\right)}$.

Define a discrete distribution over $\left\{x_{k}^{*}(i): i=\right.$ $1, \ldots, m\}$, with probability mass $q_{i}$ associated with element $i$. Now resample $m$ times from the discrete distribution to generate samples $\left\{x_{k}(i): i=1, \ldots, m\right\}$, so that for any $j, \operatorname{Prob}\left\{x_{k}(j)=x_{k}^{*}(i)\right\}=q_{i}$. It can be contended that the samples $x_{k}(i)$ are approximately distributed as the required PDF $p\left(x_{k} \mid D_{k}\right)$ [10]. Repeat this procedure until the desired number of time samples has been processed.

\subsection{Fast bootstrap technique}

The resampling method in the update process of bootstrap filter is the discrete version of inverse transform method: draw a random sample $U$ from the uniform $(0,1]$ distribution, and choose $x_{k}^{*}(M)$ for making up the posterior when

$\sum_{j=1}^{M-1} q_{j}<U \leqslant \sum_{j=1}^{M} q_{j}$.

If $m$ is large, this process takes a very long time to complete, and the problem is further exacerbated when a large number of samples are needed. A fast bootstrap algorithm was proposed by Beadle and Djuric [17] to overcome the problem. It is based on the expected number of times at which a prior sample appears in the posterior at each time instant, as described below.

With a sequence of $m$ trials, the weighted bootstrap resampling procedure can be analyzed as a sequence of Bernoulli trials. Thus, the probability of 'success' 
on a single trial (i.e., selecting an $x_{k}^{*}(M)$ ) is $p=q_{M}$, and the probability of 'failure' is $q=1-q_{M}$. The probability of the times selecting the value $x_{k}^{*}(M)$ in $m$ trials is given by the binomial distribution of order $m$. For $m$ trails, the expected number of times that any prior sample $x_{k}^{*}(i)$ appears in the posterior is $m q_{i}$. This is the key to the fast algorithm.

In the fast algorithm, at a fixed time instant $k$, pick one of the $m$ prior samples randomly from $\left\{x_{k}^{*}(i)\right\}$, say $x_{k}^{*}(M)$, assign a sampling probability of $1 / m$ for each $x_{k}^{*}(i)$, and place $\left[m q_{M}\right]$ samples into the posterior at a time, rather than only place one sample in the standard bootstrap approach. The symbol [ [ ] means the largest integer not exceeding the content. This method is repeated until a total of $m$ samples have been generated. Due to the random selection of the samples from the prior, it is possible to generate more than $m$ samples in the posterior. When this happens, the posterior sample vector is truncated to the first $m$ samples selected.

\section{Parameter estimation of SL model}

\subsection{Basic philosophy}

Regarding the unknown parameters as state variables, one can define an augmenting state vector $X$ as

$$
\begin{aligned}
X^{\mathrm{T}} & =\left\{x, \dot{x}, z, a, b, A, \beta, \gamma, n, \delta_{s}, \sigma\right\} \\
& =\left\{x_{1}, x_{2}, x_{3}, \ldots, x_{10}, x_{11}\right\} .
\end{aligned}
$$

Eqs. (5)-(8) can then be rewritten in the form of non-linear state equations:

$\dot{X}=f(X, t)$,

where

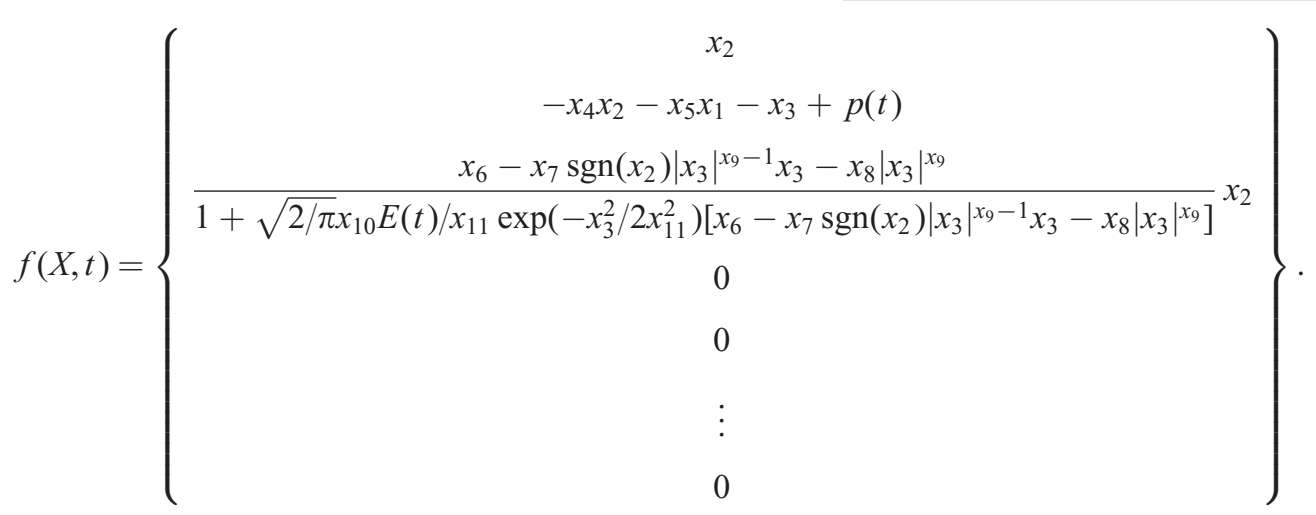

The observation equation here is expressed as

$$
Y=C X+V \text {, }
$$

where $C$ is the observation matrix and $V$ is the observation noise vector.

Utilizing the fast bootstrap filtering technique in Eqs. (19) and (20), the state vector $X(t)$ can be estimated from the input $p(t)$ and the observed output $\{Y\}_{i}$. Hence the unknown parameters are estimated simultaneously.

\subsection{Choice of initial estimates}

To start the identification algorithm, good initial estimates of parameters are needed. Here a method to decide the initial estimates of parameters is suggested. Noting the fact that $n$ is a positive real number, one can choose a suitable positive value $n_{0}$ as an initial estimate of $n$ at first. The parameters $\delta_{s}$ and $\sigma$ in this model reflect the degree of pinching and the sharpness of loop as shown in Fig. 3. It is not difficult for one to get a suitable initial value of $\delta_{s}$ and $\sigma$ according to the degree of pinching and the sharpness of loops as well as the maximum displacement and the observed dissipated energy of system. Here the corresponding system displacement $x(t)$ can be obtained by direct measurement or through integration of $\hat{\ddot{x}}(t)$, the actual observation of acceleration.

For the choice of initial estimates of $a$ and $b$, an effective method was obtained in practice by fitting a linear model to the data as follows [13]:

$$
\begin{aligned}
& \int_{0}^{T} \ddot{x}(t) x(t) \mathrm{d} t+\hat{a}_{\text {eq }} \int_{0}^{T} \dot{x}(t) x(t) \mathrm{d} t \\
& \quad+\hat{b}_{\text {eq }} \int_{0}^{T} x^{2}(t) \mathrm{d} t=\int_{0}^{T} p(t) x(t) \mathrm{d} t,
\end{aligned}
$$




$$
\begin{aligned}
& \int_{0}^{T} \ddot{x}(t) \dot{x}(t) \mathrm{d} t+\hat{a}_{\text {eq }} \int_{0}^{T} \dot{x}^{2}(t) \mathrm{d} t \\
& \quad+\hat{b}_{\text {eq }} \int_{0}^{T} x(t) \dot{x}(t) \mathrm{d} t=\int_{0}^{T} p(t) \dot{x}(t) \mathrm{d} t
\end{aligned}
$$

where $T$ is the sample length of the selected initial motion records. Defining

$\hat{\dot{x}}(t)=\int_{0}^{t} \hat{\ddot{x}}(\tau) \mathrm{d} \tau, \quad \hat{x}(t)=\int_{0}^{t} \hat{\dot{x}}(\tau) \mathrm{d} \tau$

and replacing $\ddot{x}(t), \dot{x}(t), x(t)$ by $\hat{x}(t)$ and $\hat{\dot{x}}(t), \hat{x}(t)$, respectively, one can get suitable initial estimates of $a$ and $b$ according to $\hat{a}_{\text {eq }}$ and $\hat{b}_{\text {eq }}$.

If initial estimates of $a, b, \delta_{s}, n, \sigma$, say $a_{0}, b_{0}, \delta_{s 0}, n_{0}$ and $\sigma_{0}$, are available, a method to estimate the initial values of $A, \beta$ and $\gamma$ is suggested here. According to Eqs. (6)-(9) and the assumption of $\operatorname{sgn}\left(\dot{x}_{1}\right)=\operatorname{sgn}(\dot{x})$ [5], an equation about $z$ is yielded as below

$$
\begin{aligned}
\dot{z}= & A\left(\dot{x}-\dot{x}_{2}\right)-\beta \operatorname{sgn}(\dot{x})\left(\dot{x}-\dot{x}_{2}\right) z|z|^{n-1} \\
& -\gamma\left(\dot{x}-\dot{x}_{2}\right)|z|^{n},
\end{aligned}
$$

where $\dot{x}_{2}(t)$ is defined by Eq. (7). From Eq. (22)

$$
\begin{aligned}
\overline{x \dot{z}}= & \overline{A x\left(\dot{x}-\dot{x}_{2}\right)}-\overline{\beta x \operatorname{sgn}(\dot{x})\left(\dot{x}-\dot{x}_{2}\right) z|z|^{n-1}} \\
& -\overline{\gamma x\left(\dot{x}-\dot{x}_{2}\right)|z|^{n}}, \\
\overline{\dot{x} \dot{z}}= & \overline{A \dot{x}\left(\dot{x}-\dot{x}_{2}\right)}-\overline{\beta \dot{x} \operatorname{sgn}(\dot{x})\left(\dot{x}-\dot{x}_{2}\right) z|z|^{n-1}} \\
& -\overline{\gamma \dot{x}\left(\dot{x}-\dot{x}_{2}\right)|z|^{n}}, \\
\overline{\ddot{x} \dot{z}=} & \overline{A \ddot{x}\left(\dot{x}-\dot{x}_{2}\right)}-\overline{\beta \ddot{x} \operatorname{sgn}(\dot{x})\left(\dot{x}-\dot{x}_{2}\right) z|z|^{n-1}} \\
& -\overline{\gamma \ddot{x}\left(\dot{x}-\dot{x}_{2}\right)|z|^{n}},
\end{aligned}
$$

where an overbars ${ }^{-}$denotes the time average, for example, $\bar{x}=(1 / T) \int_{0}^{T} x(t) \mathrm{d} t$. Define

$$
\begin{aligned}
& \hat{z}(t)=p(t)-\hat{\ddot{x}}(t)-a_{0} \hat{\dot{x}}(t)-b_{0} \hat{x}(t), \\
& \hat{\dot{z}}(t)=\frac{\hat{z}(t+\Delta t)-\hat{z}(t-\Delta t)}{2 \Delta t},
\end{aligned}
$$

in which $\Delta t$ is the sampling interval and $\hat{\dot{x}}(t), \hat{x}(t)$ are defined as the same as in Eqs. (21), and

$\hat{\dot{x}}_{2}=\sqrt{\frac{2}{\pi}} \frac{\delta_{s 0} \hat{E}(t)}{\sigma_{0}} \exp \left[-\frac{\hat{z}^{2}}{2 \sigma_{0}^{2}}\right] \hat{\dot{z}}$,

where $\hat{E}(t)$ is computed by

$\hat{E}(t)=\int_{0}^{t} \hat{z}(t) \mathrm{d} x=\int_{0}^{t} \hat{z}(t) \hat{\dot{x}}(t) \mathrm{d} t$.

Now replacing $z(t), \dot{z}(t), \ddot{x}(t), \dot{x}(t)$ and $x(t)$ in (23) by $\hat{z}(t), \hat{\dot{z}}(t), \hat{\ddot{x}}(t), \hat{\dot{x}}(t)$ and $\hat{x}(t)$, respectively, the initial estimates of $A, \beta$ and $\gamma$ can be obtained by solving these equations.

\section{Numerical example and discussions}

\subsection{Numerical example}

In order to validate the proposed identification method, numerical simulations were carried out for a single-degree-of-freedom hysteretic system described by Eqs. (5)-(9). The system was assumed to be subjected to the El Centro earthquake (NS, 1940), as shown in Fig. 4. The model parameters were selected to have the following values:

$a=0.5, \quad b=30, \quad A=20, \quad \beta=5$,

$\gamma=5, \quad n=2, \quad \delta_{s}=0.06, \quad \sigma=0.05$.

Eqs. (5)-(9) with these parameters were solved numerically by using the fourth-order Runge-Kutta

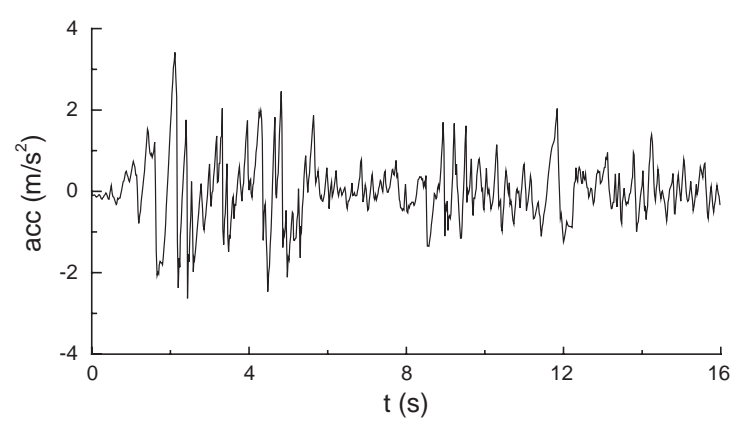

Fig. 4. El Centro earthquake (NS, 1940). 


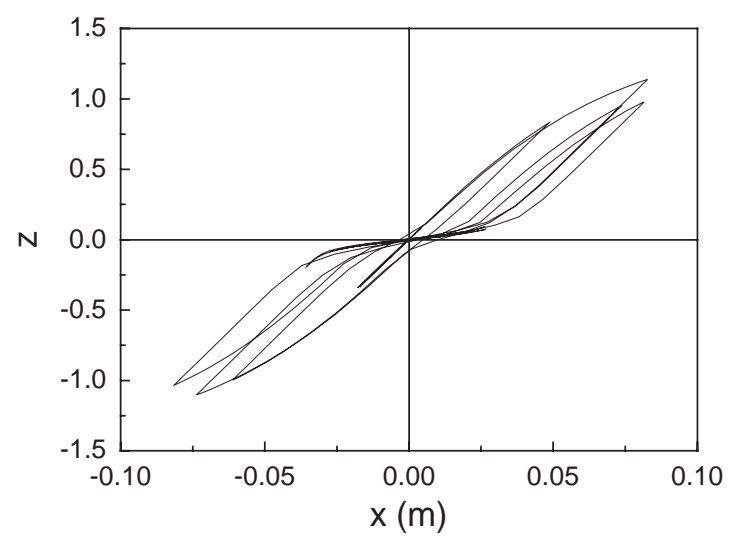

Fig. 5. Hysteresis loops.

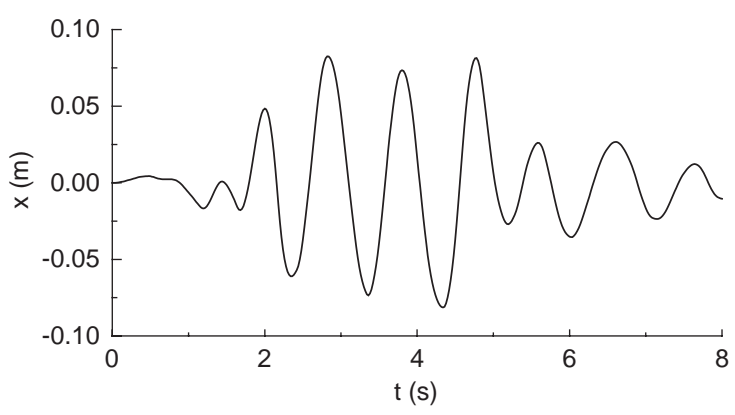

Fig. 6. Displacement response.

method with a time step of $0.02 \mathrm{~s}$ and the hysteresis loops are shown in Fig. 5. The obtained displacement $x(t)$ (as shown in Fig. 6) contaminated by noise $v_{k}$ was used as the experimental measurement. The observation equation (20) is then given as follows:

$$
\begin{aligned}
y_{k}= & {\left[\begin{array}{lllllllllll}
1 & 0 & 0 & 0 & 0 & 0 & 0 & 0 & 0 & 0 & 0
\end{array}\right] } \\
& \times\{X\}_{k}+v_{k} .
\end{aligned}
$$

Here the noise $v_{k}$ is assumed to be a zero-mean random process, which has the Student's $t$ distribution with four degrees of freedom scaled according to the standard deviation of the principle noise component. This distribution has heavier tails than the Gaussian distribution so that exceptionally large measurement errors may occur, i.e., it accommodates the outliers [18]. Thus the PDF of the measurement error is modeled as

$p(v) \propto\left(n+v^{2} / s^{2}\right)^{-(n+1) / 2}$,

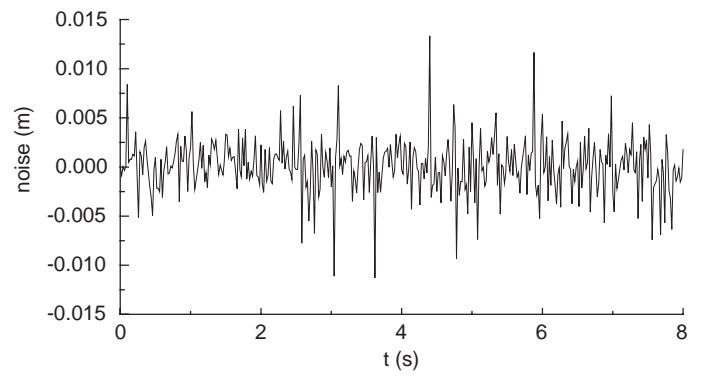

Fig. 7. A noise sample with $t$ distribution.

where the degree of freedom $n=4$ and the scale $s=0.002$. Since the update weights are normalized as shown in Eq. (16), the PDF of the measurement error need only be specified to proportionality. Fig. 7 depicts a noise sample simulated from this distribution.

The initial conditions for this analysis were estimated according to the method proposed in this paper and given in Table 1. The distributions of all initial estimations are assumed to be Gaussian, and the total number of sample realizations is $m=1000$. It should be noted that the assumption of Gaussian distributions here is not a requirement.

The parameters are estimated by the identification method proposed in this paper, and the estimated results are given in Table 2. The distribution of process noise $p_{w}\left(w_{k}\right)$ is assumed to be Gaussian with zero mean and a standard deviation of 0.3 . Figs. $8-15$ show the time histories of means of the estimated parameters. It can be seen that the parameters converge to good estimated values.

\subsection{Discussions}

(1) A problem encountered in applying the proposed method is the handling of low probability events known as outliers. At a particular time instant $k$, the product of $m q_{i}$ may be less than one, then those $x_{k}^{*}(i)$ in the prior cannot generate any samples into the posterior using the $[\cdot]$ criterion. The bootstrap, on the other hand, guarantees each prior sample a chance, no matter how small, of being selected in the posterior. To overcome this problem, two ways were suggested in [17]. One is to choose an $m$ large enough so that $\left[m q_{i}\right] \geqslant 1$ for almost every $x_{k}^{*}(i)$ in the prior. However, no matter how large $m$ is selected, there can be 
Table 1

Initial conditions

\begin{tabular}{|c|c|c|c|c|c|c|c|c|c|c|c|}
\hline Variable & $x$ & $\dot{x}$ & $z$ & $a$ & $b$ & $A$ & $\beta$ & $\gamma$ & $n$ & $\delta_{S}$ & $\sigma$ \\
\hline $\bar{x}\left(t_{0} \mid t_{0}\right)$ & 0 & 0 & 0 & 0.28 & 35 & 30 & 6.5 & 6.8 & 1.8 & 0.03 & 0.08 \\
\hline$P\left(t_{0} \mid t_{0}\right)$ & 0.001 & 0.001 & 0.001 & 0.1 & 80 & 150 & 20 & 20 & 0.01 & 0.01 & 0.01 \\
\hline
\end{tabular}

Table 2

Identified parameters

\begin{tabular}{lllllllll}
\hline Parameter & $a$ & $b$ & $A$ & $\beta$ & $\gamma$ & $n$ & $\delta_{s}$ \\
\hline Exact value & 0.5 & 30 & 20 & 5 & 5 & 2 & 0.06 \\
Identified value & 0.5674 & 32.55 & 17.7649 & 5.778 & 5.5523 & 1.8914 & 0.0617 & 0.05 \\
\hline
\end{tabular}

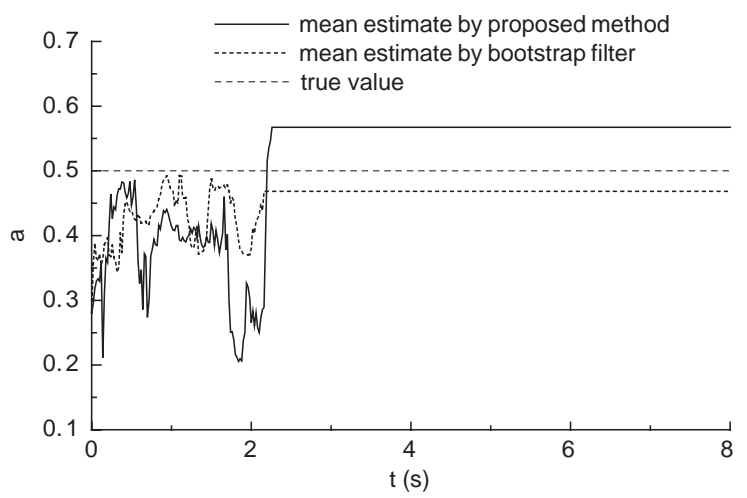

Fig. 8. Estimate of parameter $a$.

outliers still ignored. Hence, given the same set of prior samples, the low probability states are underrepresented in the posterior. Another solution is to use $\max \left(\left[m q_{M}\right], 1\right)$ as the number of times of including the selected value $x_{k}^{*}(M)$ in the posterior. Thereby, even those values of outliers can be selected at least once into the posterior. However, there is a danger here because if $m$ is too small, some outliers can be over-represented in the posterior by selecting them much more often than they should be. We tried this method in numerical simulations, but the results were not good. The estimated values fluctuate severely and sometimes become unstable as shown in Fig. 16.

The method we used in this paper to overcome the above problem is a combination approach. In this method, each prior sample $x_{k}^{*}(i)$ with a $q_{i}$ such that $\left[m q_{i}\right]=0$, is "tagged" when it is selected the first time, so that it will not be selected again at a particular time

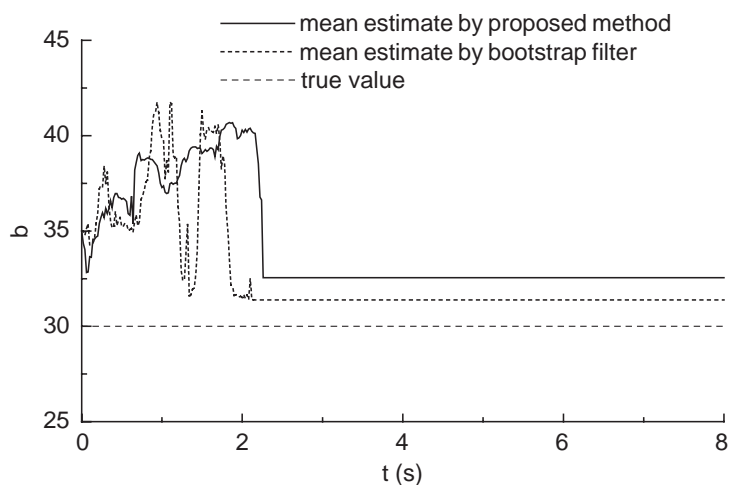

Fig. 9. Estimate of parameter $b$.

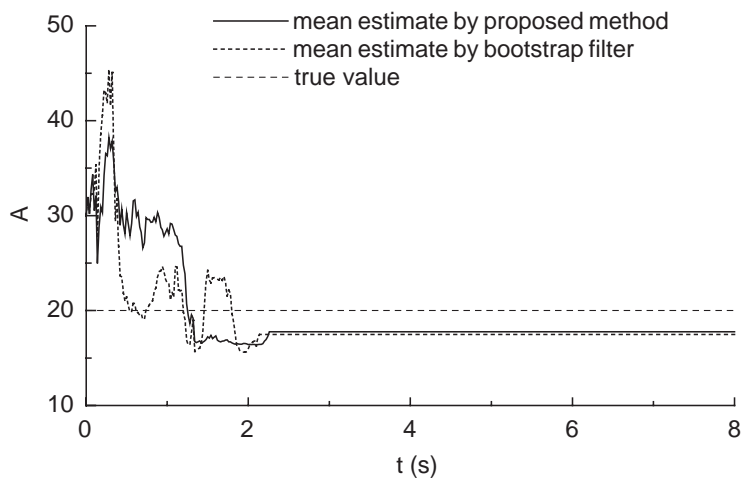

Fig. 10. Estimate of parameter $A$.

step. This guarantees that each sample from the prior with a probability less than $1 / m$ can only appear at most once in the posterior. With this approach, good estimated values were obtained (Figs. 8-15). 


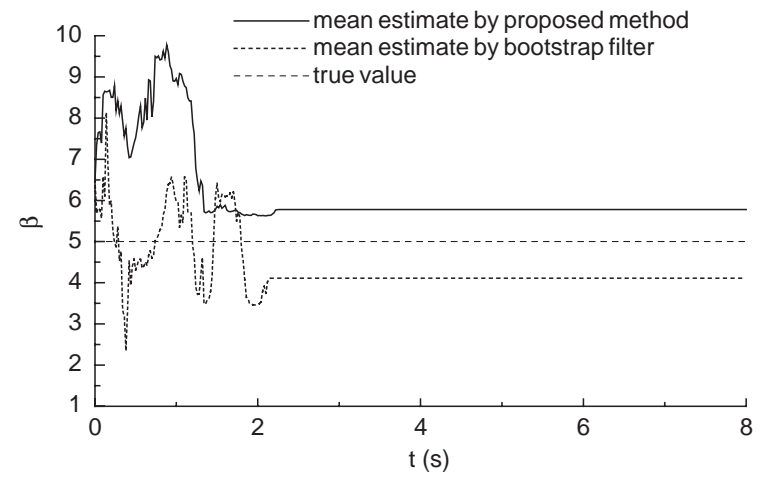

Fig. 11. Estimate of parameter $\beta$.

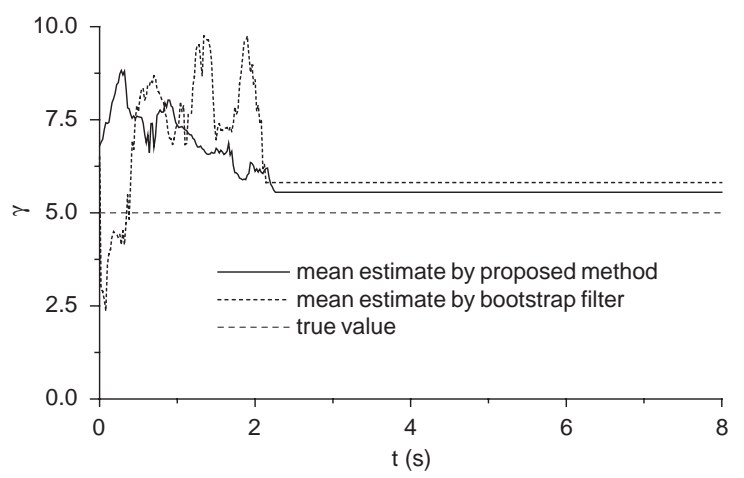

Fig. 12. Estimate of parameter $\gamma$.

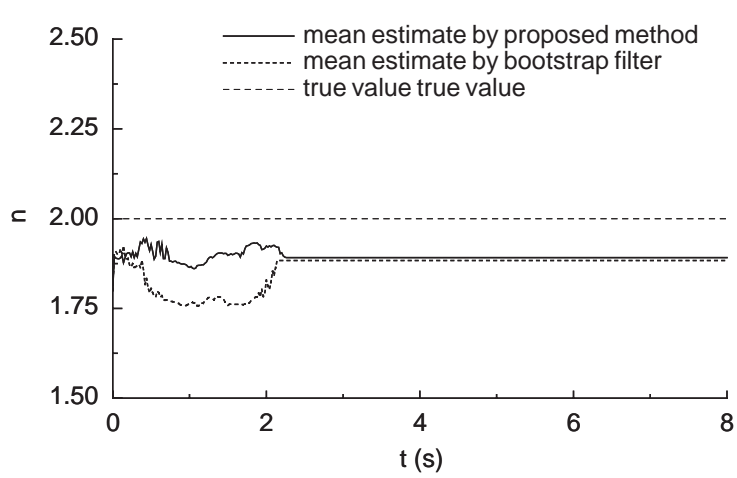

Fig. 13. Estimate of parameter $n$.

(2) The estimation results using standard bootstrap method are also given in Figs. 8-15 to compare the performance of two methods. While the proposed algorithm provides parameter estimates as good as the standard method, it is faster than the standard boot-

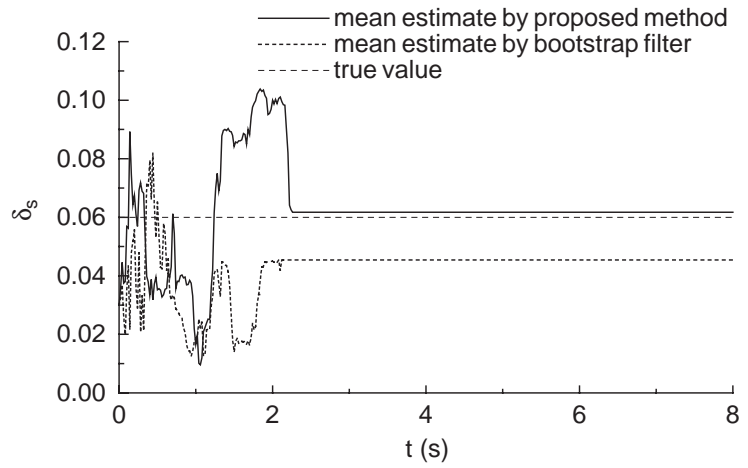

Fig. 14. Estimate of parameter $\delta_{s}$.

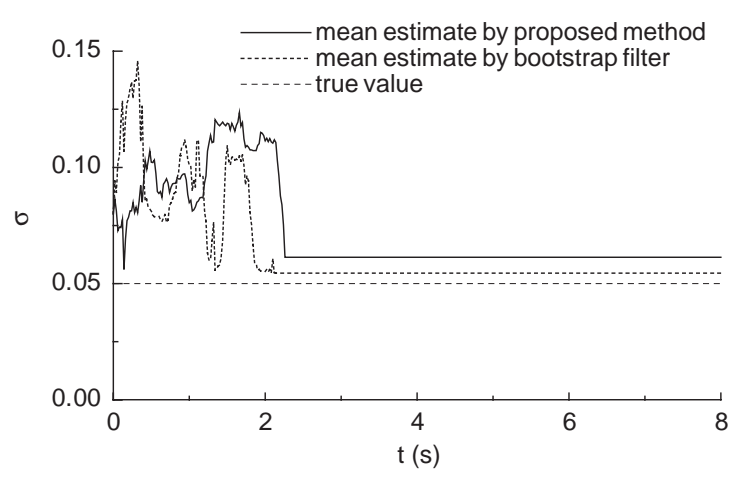

Fig. 15. Estimate of parameter $\sigma$.

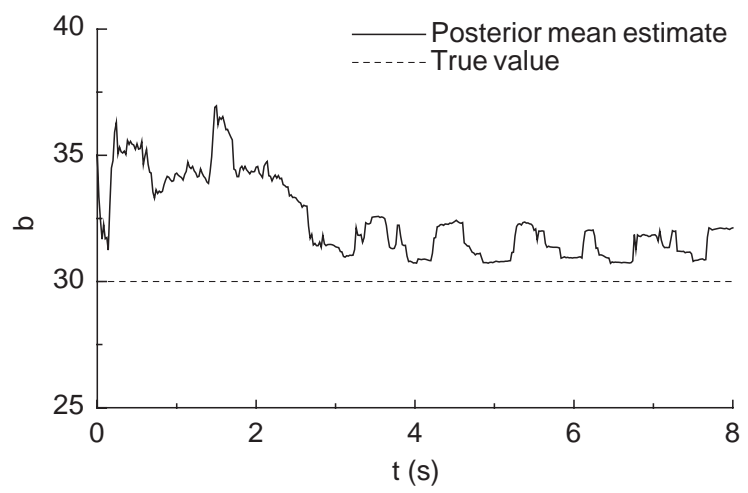

Fig. 16. Estimate of parameter $b$ using $\max \left(\left[m q_{M}\right], 1\right)$ criterion.

strap method by a factor of approximately 6 for the period from beginning to the convergence. And this effect will be more significant with the increasing of sample size. However, the reduction of the computational time is less obvious after the convergence takes 

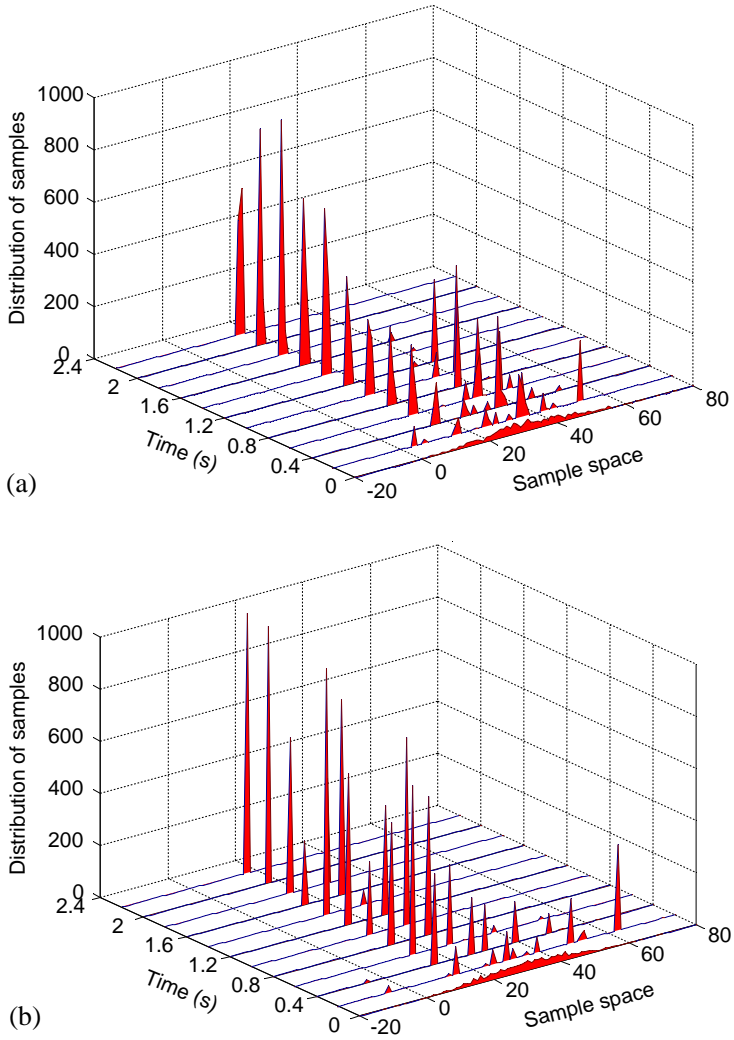

Fig. 17. Sample distributions of parameter $A$ at different time step: (a) proposed method; (b) standard bootstrap filter-based method.

place. The reason is that the variance of the prior samples decreases after the convergence happens, samples in prior will tend to a same weighted probability of $1 / \mathrm{m}$. Hence, the resampling procedure in the fast algorithm is almost the same as that in the standard bootstrap method, and the execution time cannot be reduced obviously.

(3) Fig. 17 shows the sample distributions of parameter $A$ at different time step computed with different algorithms. It can be seen clearly that the sample distribution of parameter $A$ is not Gaussian due to the non-linearity of system even if the distribution of initial estimation is assumed to be Gaussian.

\section{Conclusion}

In this paper, a parameter estimation method based on a fast Bayesian bootstrap filter is proposed for non-linear hysteretic systems with slip. The method requires much less computation time than standard bootstrap filter based approach, while maintaining the comparable accuracy. It is especially suitable for real time applications. The decrease in computation time is achieved by resampling the posterior in groups instead of one sample at a time. Further work on this technique will be carried on to investigate its applicability to other non-linear models, especially to those with time-varying parameters.

\section{References}

[1] Y. Suzuki, R. Minai, Application of stochastic differential equations to seismic reliability analysis of hysteretic structures, Probab. Eng. Mech. 3 (1988) 43-52.

[2] R. Bouc, Forced vibration of mechanical system with hysteresis, Proceedings of the 4th Conference on Nonlinear Oscillations, Prague, Czechoslovakia, 1967, p. 315.

[3] Y.K. Wen, Method for random vibration of hysteretic systems, J. Eng. Mech. 102 (1976) 249-263.

[4] T.T. Baber, Y.K. Wen, Random vibration of hysteretic degrading systems, J. Eng. Mech. 107 (1981) 1069-1089.

[5] T.T. Baber, M.N. Noori, Random vibration of degrading pinching systems, J. Eng. Mech. 111 (1985) 1010-1026.

[6] S.F. Masri, T.K. Caughey, A nonparametric identification technique for nonlinear dynamic problems, J. Appl. Mech. 46 (1979) 433-447.

[7] Y.Q. Ni, J.M. Ko, C.W. Wong, Nonparametric identification of nonlinear hysteretic systems, J. Eng. Mech. 125 (1999) 206-215.

[8] M. Hoshiya, E. Saito, Structural identification by extended Kalman filter, J. Eng. Mech. 110 (1984) 1757-1770.

[9] C.-B. Yun, M. Shinozuka, Identification of nonlinear structural dynamic systems, J. Struct. Mech. 8 (1980) 187-203.

[10] N.J. Gordon, D.J. Salmond, A.F.M. Smith, Novel approach to nonlinear/non-Gaussian Bayesian state estimation, IEE Proc.-F 140 (1993) 107-113.

[11] M. Hoshiya, O. Maruyama, State estimation of conditional non-Gaussian random fields by BF/MCF, Proceedings of U.S. -Japan Cooperative Research on Urban Earthquake Disaster Mitigation: U.S.-Japan Joint Workshop and Third Grantees Meeting, University of Washington, Seattle, Washington, USA, 2001, pp. 226-236.

[12] R.H. Sues, S.T. Mau, Y.K. Wen, Systems identification of degrading hysteretic restoring forces, J. Eng. Mech. 114 (1988) 833-846.

[13] M. Yar, J.K. Hammond, Parameter estimation for hysteretic systems, J. Sound Vib. 117 (1987) 161-172.

[14] A.G. Chassiakos, S.F. Masri, A.W. Smyth, T.K. Caughey, On-line identification of hysteretic systems, J. Appl. Mech. 65 (1998) 194-203. 
[15] J.W. Lin, R. Betti, A.W. Smyth, R.W. Longman, On-line identification of non-linear hysteretic structural systems using a variable trace approach, Earthquake Eng. Struct. Dyn. 30 (2001) 1279-1303.

[16] S.J. Li, Y. Suzuki, M. Noori, Identification of hysteretic structural systems with slip by Bayesian method and bootstrap filter, in: W.Q. Zhu, G.Q. Cai, R.C. Zhang (Eds.), Advances in Stochastic Structural Dynamics, CRC Press, Boca Raton, FL, 2003, pp. 333-344.
[17] E.R. Beadle, P.M. Djuric, A fast weighted Bayesian bootstrap filter for nonlinear model state estimation, IEEE Trans. Aerosp. Electron. Syst. 33 (1997) 338-343.

[18] N. Gordon, D. Salmond, C. Ewing, Bayesian state estimation for tracking and guidance using the bootstrap filter, J. Guidance, Control Dyn. 18 (1995) 1434-1443. 